
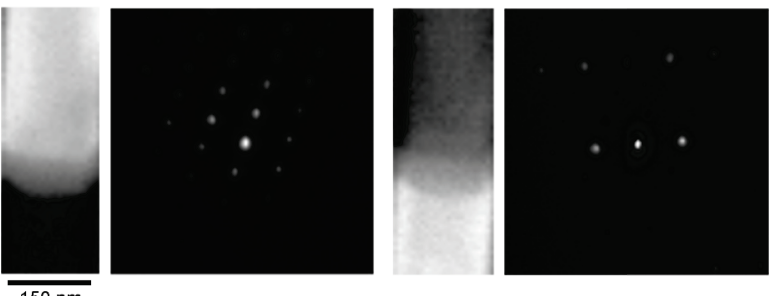

Figure 1. Component patterns with their spatial loadings across an inclined twin boundary in a GaAs nanowire decomposed from scanning precession electron diffraction data by using non-negative matrix factorisation. Overlapping bright regions in loading maps indicate where mixed patterns were separated.

Keywords: Orientation Imaging, Crystallographic Mapping, Precession Electron Diffraction, Machine Learning, Multivariate Statistical Analysis

\section{MS27-P9 Enhanced technique for characterization of strain distribution in crystal from EBSD patterns}

Vadym Khomenko ${ }^{1}$

1. Yuriy Fedkovych Chernivtsi National University, Chernivtsi, Ukraine

email: primaldarkness@mail.ru

Electron backscattering diffraction method (Kikuchi method) is used for determination of crystal orientation, phase, strain and stress state of crystals. Elastic strains caused by the distortion of crystal structure change the interplanar distances [1]. The last ones reveal themselves in the changing of intensity distribution onto the Kikuchi pattern to have been measured. Due to the high spatial resolution and high locality the EBSD method can be used for studying of highly inhomogeneous samples. Weld joints of $\mathrm{NiCrFe}$ and artificial diamond crystals are these kinds of materials. Kikuchi patterns were obtained by using scanning electron microscope "Zeiss" Evo-50 with CCD detector

The enhanced technique which combines two-dimensional Fourier transformation of Kikuchi pattern as whole and analysis of intensity profiles of separate Kikuchi bands was developed. The strain tensor determination was performed using the new approach which is based onto the local cross-correlation of intensity peaks of multi-beam areas.

Approbation of developed technique was carried out on two diamonds samples: one of them was obtained by temperature gradient method in $\mathrm{Fe}-\mathrm{Al}-\mathrm{C}$ system and second - in $\mathrm{Mg}-\mathrm{C}+$ bor system by growth method on diamond single crystal synthesized in $\mathrm{Ni}-\mathrm{Mn}-\mathrm{C}$ system. As a result characteristic surfaces of strain tensor were constructed for local areas, which correspond to different crystalline blocks (Fig. 1).

Next, the samples of weld joint of $\mathrm{NiCrFe}$ nickel alloy with crack have been researched for the purpose of establishment of probable causes of crack formation. In addition to Kikuchi patterns the distribution of chemical composition was obtained by means of X-ray microanalysis. The features of strain distribution around crack were established. Particularly, it was demonstrated that maximal strain values are spreaded along the low angle boundaries of subgrains.

1. Fodchuk I. Determination of structural inhomogeneity of synthesized diamonds by backscattering electron diffraction / I.Fodchuk, S. Balovsyak, M. Borcha, Ya. Garabazhiv, V. Tkach // Phys. Status Solidi A. - 2011. - Vol. 208, № 11. - P. 2591-2596. 

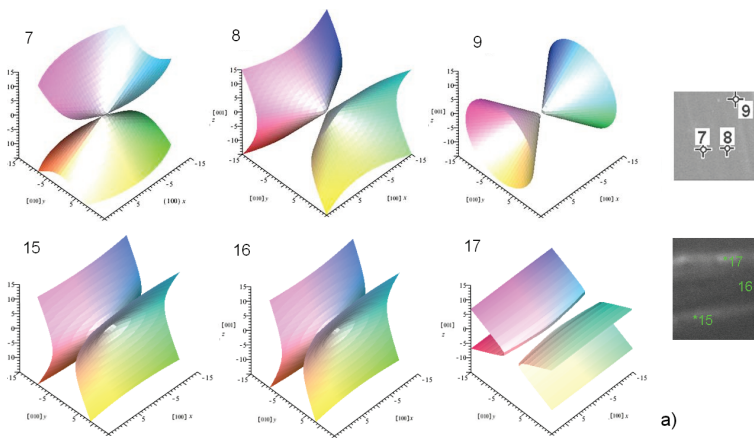

Figure 1. Characteristic surfaces of strain tensor (a), which corresponds to different local areas (labaled by numbers) of diamond crystal (b)

Keywords: EBSD, strain measurement, strain tensor, diamond crystals, weld joints

\section{MS28. Charge density studies}

Chairs: Anders Madsen, Simon Coles

\section{MS28-P1 Lone electron pair dispersion - experimental charge density study of cubic arsenic(III) oxide}

Piotr A. Gunka ${ }^{1}$, Zygmunt Gontarz ${ }^{1}$, Janusz Zachara ${ }^{1}$

1. Warsaw University of Technology, Faculty of Chemistry

email: piogun@ch.pw.edu.pl

The first experimental charge density study of arsenolite, cubic polymorph of arsenic(III) oxide, extended by periodic DFT calculations is reported. The presence of weak As ‥O interactions is confirmed and their topological characterization based on experimental electron density is provided utilizing both the Quantum Theory of Atoms in Molecules (QTAIM) and non-covalent interactions descriptor based on reduced density gradient (NCI-RDG). Spatial dispersion of arsenic lone electron pair (LEP) into three domains is observed in the Laplacian of electron density as well as in electron localization function (ELF) for the first time. The domains are located trans with respect to the primary As-O bonds as evidenced by the analysis of ELF. This could be related to the formation of these chemical bonds and/or to the tetrahedral clustering of arsenic atoms. Similar clustering has been observed in $\mathrm{Sb}(\mathrm{III})$ and $\mathrm{Bi}(\mathrm{III})$ oxysalts, suggesting LEPs may play significant role in this phenomenon. The dispersion of LEPs may be a more general effect for heavy elements and the issue should be investigated thoroughly to understand its origin and structural consequences.

Authors thank National Science Centre for financial support of this work (DEC2012/05/N/ST5/00283). 